\title{
Numerical simulations and experiments of beam-target interaction for multipulse bremsstrahlung converter applications
}

\author{
Hai-jun Yu, Jun Zhu, Nan Chen, Yuan Wang, Zhen-tao Zhang, Xiao-guo Jiang, \\ Wen-hua Dai, and Cheng-jun Liu \\ Institute of Fluid Physics, CAEP, Postcode 621900, Mianyang, Sichuan, China
}

(Received 23 October 2011; published 12 June 2012)

\begin{abstract}
The typical response of an x-ray converter impacted by an intense relativistic electron beam is vaporization and rapid expansion. In the multipulse electron beam/target interaction process, the target expands, the density of material decreases, eventually affecting the x-ray output of the system. We report the relation between target material thickness with x-ray doses, and the design of a distributed target for multipulse beam/target interaction application. For a single pulse, we simulated and measured the x-ray doses and spot size on the distributed target, and compared them to similar results made by the standard converter target. For multipulses of microsecond time scale, we measured the change of the distributed target material density. The results indicate that the multipulse converter target design is feasible.
\end{abstract}

DOI: 10.1103/PhysRevSTAB.15.060401

PACS numbers: 29.27.-a, 87.59.B-, 52.59.Px

\section{INTRODUCTION}

Linear induction accelerators (LIA), such as AIRIX, DARHT-I, and DRAGON-I, produce intense x-ray pulse by focusing a short pulse of high current electron beam onto a high $Z$ material target (bremsstrahlung converter target) [1-6]. The electrons deposit enormous amounts of energy into the target in a small volume during a very short time period $(\sim 60 \mathrm{~ns})$, raise the temperature of the material up to more than $2 \mathrm{eV}$ [7], and lead to a violent hydrodynamic motion inside the target. The converter target material may vaporize and rapidly disperse. These machines are single pulse machines providing time for target replacement between shots. But it is completely different for the multipulse $\mathrm{x}$-ray machines such as DARHT-II; there is no time for target replacement during the multipulse operation on a $\mu$ s time scale; it may result in a decreased material density for succeeding pulses. As a result, the efficiency of the latter x-ray production may decrease, and the dose of the latter $\mathrm{x}$-ray pulses may also decrease.

In this paper, the relation of $\mathrm{x}$-ray dose with target material effective thickness (we use the "equivalent thickness" to define the net material thickness of the target) for single pulse electron is first simulated; the minimal effective thickness for providing enough x-ray dose is obtained. We also designed a distributed target for multipulse operation and compared the $\mathrm{x}$-ray dose distribution between the distributed target and the standard target with numerical simulations and experiment measurement. A soft x-ray generator will be used to backlight the target after a speci-

Published by the American Physical Society under the terms of the Creative Commons Attribution 3.0 License. Further distribution of this work must maintain attribution to the author(s) and the published article's title, journal citation, and DOI. fied delay. By measuring the intensity of the transmitted $x$ rays, we are able to determine the relative change of the target material density on the microsecond time scale (the time scale for multipulse operation).

\section{NUMERICAL SIMULATIONS OF TARGET EFFECTIVE THICKNESS VERSUS X-RAY DOSE FOR SINGLE PULSE OPERATION}

The high current electron beam interacts with bremsstrahlung target to produce high dose $\mathrm{x}$ ray. The $\mathrm{x}$-ray dose depends on the electron energy, the electron beam current, target material $(Z)$, and effective target thickness. Material of high atomic number $Z$ (tantalum) is often chosen in order to improve the $\mathrm{x}$-ray dose. The $\mathrm{x}$-ray dose solely depends on the target thickness for a given electron beam and target material (Ta). We simulate the x-ray dose production at one meter as a function of tantalum target thickness for a definite single pulse electron current by

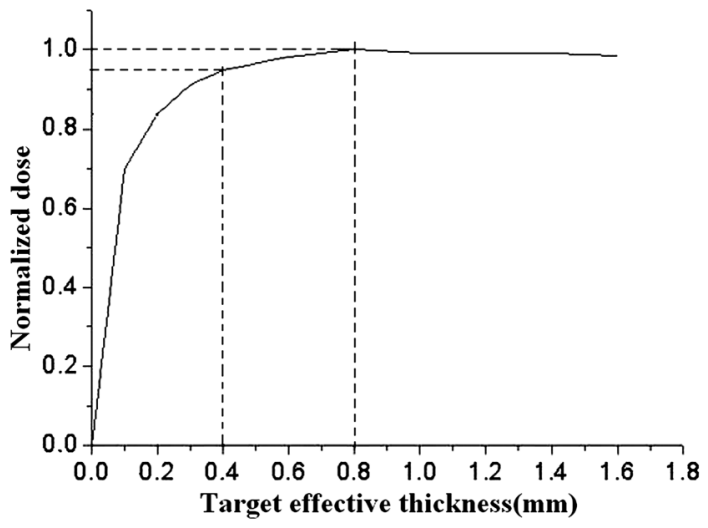

FIG. 1. Normalized x-ray dose depends on the target effective thickness. 
using the Monte-Carlo code EGS4 [8]. The electron beam parameters are obtained from DRAGON-I LIA as following: the electron current $2.5 \mathrm{kA}$, the electron energy $\sim 19 \mathrm{MeV}$, and the beam pulse width $\sim 70 \mathrm{~ns}$. The normalized $x$-ray dose near $0^{\circ}$ forward scattering dependence on the tantalum target thickness is shown in Fig. 1. The figure shows that the x-ray dose exceeds $90 \%$ of the maximal dose when the target thickness is larger than $0.4 \mathrm{~mm}$. Above this value, small dose variations occur for relatively large thickness changes. Accordingly, we can conclude that the $\mathrm{x}$-ray dose will satisfy the request of the $\mathrm{x}$-ray machine for single electron pulse or multipulse operation if the target effective thickness is not less than $0.4 \mathrm{~mm}$.

\section{COMPARISON OF DIFFERENT KINDS OF CONVERTER TARGETS FOR SINGLE PULSE OPERATION}

We proposed a multipulse converter design which uses $1.2 \mathrm{~mm}$ thick tantalum foils evenly distributed over $1 \mathrm{~cm}$. The design aims are to decrease the energy deposition density in the converter target and reduce destruction of target material in order to satisfy the multipulse demand [9].

The configuration drawing of the distributed target is described in Fig. 2. We simulated the normalized angle distribution, and measured the x-ray doses and spots of two kinds of converter targets, standard and distributed target,

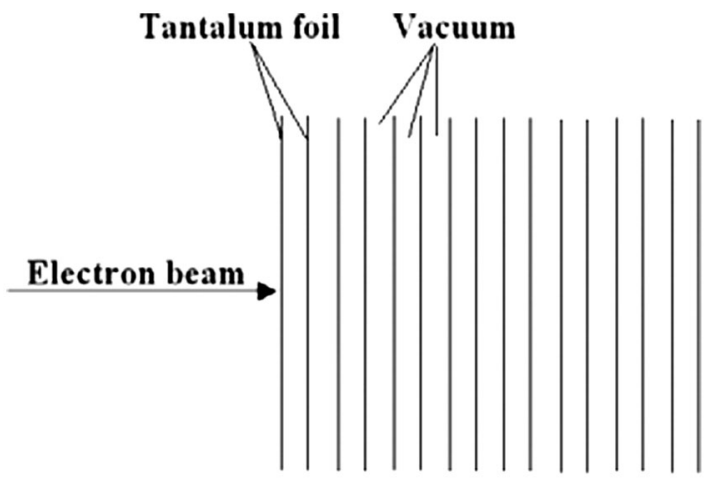

FIG. 2. Configuration of the distributed target.

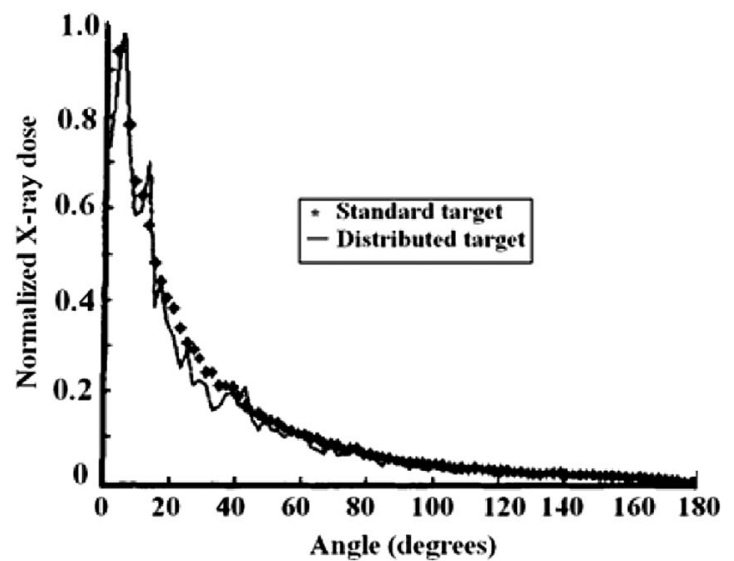

(a)

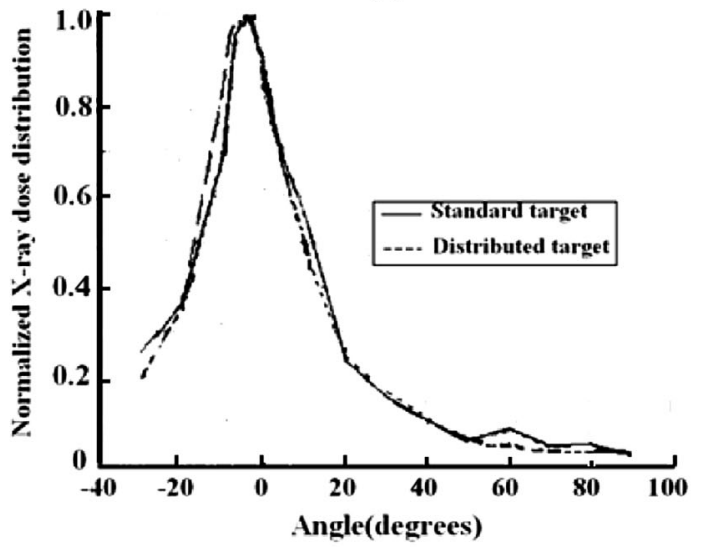

(b)

FIG. 3. Comparison of normalized x-ray dose. (a) Numerical simulation. (b) Experiment data.

for a single electron pulse, respectively. Figure 3 shows the comparison of normalized angle distribution for numerical simulation and the experiment measurement, respectively. The $\mathrm{x}$-ray spots of different experiments are given in Table I. The electron beam is produced from DRAGON-I LIA.

Figure 3 and Table I show that the distributed target can generate the similar dose after interaction with single pulse electron beam, and the x-ray spots are also stable and satisfy the demand of the single pulse electron beam operation.

TABLE I. Electron beam and x-ray parameters of DRAGON-I LIA for distributed target.

\begin{tabular}{lcccc}
\hline \hline $\begin{array}{l}\text { Experiment } \\
\text { number }\end{array}$ & $\begin{array}{c}\text { Electron } \\
\text { current (kA) }\end{array}$ & $\begin{array}{c}\text { Electron } \\
\text { energy (MeV) }\end{array}$ & $\begin{array}{c}\text { The x-ray dose } \\
\text { @ 1 m (Gy) }\end{array}$ & $\begin{array}{c}\text { X-ray spot diameter } \\
(\mathrm{FWHM})(\mathrm{mm})\end{array}$ \\
\hline 4562 & 2.54 & 19.1 & 4.03 & 1.46 \\
4563 & 2.55 & 19.1 & 3.79 & 1.50 \\
4564 & 2.57 & 19.2 & 4.06 & 1.45 \\
4565 & 2.57 & 19.2 & 3.86 & 1.46 \\
4566 & 2.54 & 19.1 & 4.10 & 1.30 \\
\hline \hline
\end{tabular}




\section{MEASUREMENT OF TARGET MATERIAL DENSITY ON THE MULTIPULSE OPERATION TIME SCALE}

We designed the experiment layout by using a soft x-ray generator system in order to measure the target density change during the multipulse operation time scale $(\sim \mu \mathrm{s})$ after target interacting with the single pulse electron beam. The design principle of the experimental system is as follows: the electron beam from DRAGON- I LIA interacts with the distributed target and generates a hard $\mathrm{x}$ ray; a soft $\mathrm{x}$ ray crosses the target and enters a fast scintillator. The fast scintillator is used as a convertor which converts the soft $x$ ray into visible light, and the visible images can be obtained from a gated intensified charge coupled device camera. The material density pattern in the distributed target is given by the shades of grey. The experiment layout of target density measurement is given in Fig. 4; the target surface was placed at an angle of $45^{\circ}$ with the beam axis. The soft x-ray generator system was set to fire $1 \mu \mathrm{s}$ after the beam impact on the target. The converter target is the distributed one of which the effective thickness is chosen as $0.4 \mathrm{~mm}$ considering the soft $\mathrm{x}$-ray penetrability and requirement of multipulse $x$-ray dose. The parameters of soft x-ray generator are voltage $450 \mathrm{kV}$, pulse width $25 \mathrm{~ns}$, x-ray spot diameter 1-4 mm, dose @ $1 \mathrm{~m} 2.1 \times 10^{-4}$ Gy.

Figure 5 is the grey image comparison of the distributed target after beam-target interaction. Figure 5(a) shows the grey image taken $\sim 1 \mu \mathrm{s}$ after the beam-target interaction. Figure 5(b) shows the grey image taken a long time after the beam-target interaction, and the actual beam-target interaction zone has become a hole. We measured the grey value of Figs. 5(a) and 5(b), respectively, and show them in Fig. 6.

The black line in Fig. 6 corresponds to Fig. 5(b). The grey shade value increases from 3200 to 4300 , indicating

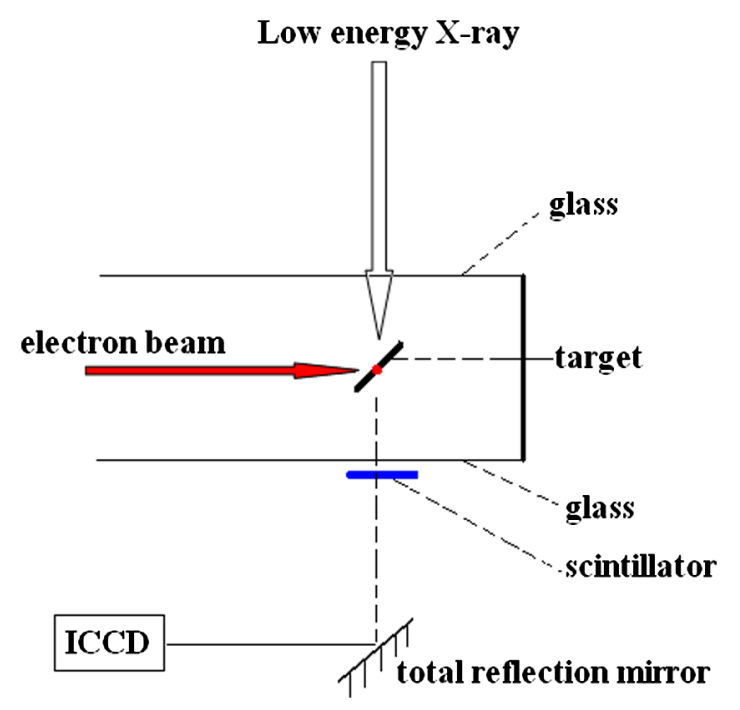

FIG. 4. Layout of the soft x-ray measurement of the converter target density.

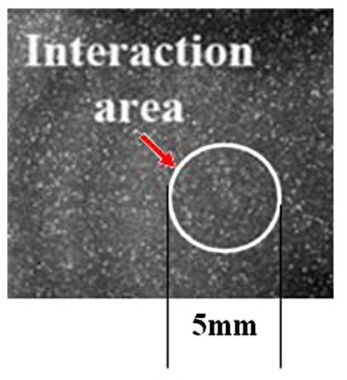

(a)



(b)
FIG. 5. Comparison of grey intensity after beam-target interaction: (a) $\sim 1 \mu$ s delay, (b) long time delay.

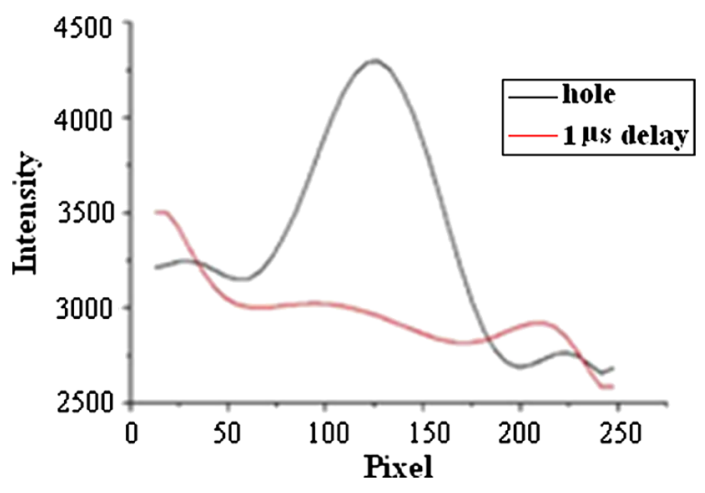

FIG. 6. Curve of grey intensity versus pixel (1 pixel $=$ $0.02 \mathrm{~mm}$ ).

that the beam produced a hole in the target. The red line stands for the grey shade change of the target $1 \mu \mathrm{s}$ after beam-target interaction [Fig. 5(a)]; it remains almost constant over 100 pixels (from 50th to 150th point in Fig. 6), the maximum change is less than $7 \%$. This indicates that over $\sim 1 \mu \mathrm{s}$ the target density undergoes a limited decrease, so the x-ray dose produced by this kind of target can satisfy the dosage requirement for the multipulse operation time scale, as confirmed by the experiment results in Table I.

\section{SUMMARY}

One important question for the multipulse electron beam converter target is how to keep enough target material and make the target density almost unchanged. In this paper we simulated the relation of $\mathrm{x}$-ray dose with target material effective thickness for single pulse operation, and obtained the minimal effective thickness. The distributed target for multipulse application is designed and tested, and the $\mathrm{x}$-ray dose and the spot produced by the distributed target are the same as the standard target ones. We measured the change of the distributed target density over the multipulse operation time scale $(\sim 1 \mu \mathrm{s})$ by using the low energy x-ray system; the experiment results show that the target material density is almost unchanged and the target material should satisfy the x-ray dose requirement for multipulse operation. 
We still need to do multipulse experiments that will test whether the distributed target can produce spot sizes small enough for the succeeding pulses.

\section{ACKNOWLEDGMENTS}

The authors would like to acknowledge valuable discussions with Professor Jinshui Shi for target density measurement, and Dr. Shaoheng Wang for the suggestions and help in the x-ray measurement, and acknowledge the DRAGON-I operation team's effort. This work is supported by National defense fundamental research project.

[1] E. Merle, R. Boivinet, and M. Mouillet, in Proceedings of 1999 Particle Accelerator Conference, New York, 1999 (IEEE, New York, 1999), p. 3260.
[2] T. J. T. Kwan, C. M. Snell, and P. J. Christensen, Phys. Plasmas 7, 2215 (2000).

[3] T. J. T. Kwan, Los Alamos National Laboratory Report No. LA-UR-98-4802, 1998.

[4] S. Sampayan, R. Buckles, and G. Caporaso, in Proceedings of 2001 Particle Accelerator Conference, Chicago, IL, 2001 (IEEE, Piscataway, NJ, 2001), p. 330.

[5] Yu-J. Chen, L. R. Bertolini, G. J. Caporaso et al., in Proceedings of the Interaction Linac Conference, Korea, 2002 (Pohang Accelerator Laboratory, Pohang, Korea, 2002), p. 16.

[6] B.-N. Ding, J.-J. Deng, and H.-C. Wang, High Energy Phys. Nucl. Phys. 29, 604 (2005).

[7] J. Zhu, H.-J. Yu, X.-G. Jiang, N. Chen, and Y. Wang, IEEE Trans. Plasma Sci. 38, 2873 (2010).

[8] W. R. Nelson, H. Hirayama, and D. W. O. Rogers, Report No. SLAC265, 1985.

[9] J. Zhu, L.-W. Zhang, J.-D. Long, J. Li, H.-J. Yu, and J.-S. Shi, High Power Laser Part. Beams 17, 1581 (2005). 